TRANSACTIONS OF THE

AMERICAN MATHEMATICAL SOCIETY

Volume 359, Number 4, April 2007, Pages 1875-1888

S 0002-9947(06)04197-3

Article electronically published on November 22, 2006

\title{
THE SEMICLASSICAL STRUCTURE OF LOW-ENERGY STATES IN THE PRESENCE OF A MAGNETIC FIELD
}

\author{
DAVID BORTHWICK AND ALEJANDRO URIBE
}

\begin{abstract}
We consider a compact Riemannian manifold with a Hermitian line bundle whose curvature is non-degenerate. The Laplacian acting on high tensor powers (the semiclassical regime) of the bundle exhibits a cluster of low-energy states. We demonstrate that the orthogonal projectors onto these states are the Fourier components of an operator with the structure of the Szegö projector, i.e. a Fourier integral operator of Hermite type. This result yields semiclassical asymptotics for the low-energy eigenstates.
\end{abstract}

\section{INTRODUCTION}

Let $(X, g)$ be a compact Riemannian manifold and $L \rightarrow X$ a Hermitian line bundle with connection, $\nabla$, which we think of as a magnetic potential. The curvature (or field strength) of $\nabla$ will be denoted by $\omega$, a closed two-form on $X$. In this paper we will work under the assumption that

$$
\omega \text { is non-degenerate. }
$$

This assumption implies that there is a bundle automorphism, $K: T X \rightarrow T X$, such that

$$
\forall x \in X \quad u, v \in T_{x} X \quad g_{x}(u, K(v))=\omega_{x}(u, v) .
$$

It is easy to check that $K$ is skew adjoint with respect to $g$, and therefore for each $x \in X$ the eigenvalues of $K_{x}$ can be written in the form $\pm i \kappa_{j}(x)$ where $\kappa_{j}(x)>0$, $j=1, \ldots, n$, and the dimension of $X$ is denoted $2 n$. Introduce the function

$$
\operatorname{Tr}^{+} K(x)=\sum_{j=1}^{n} \kappa_{j}(x) .
$$

It is easy to see that this function is smooth, although the individual $\kappa_{j}$ may cross. For future reference we also introduce here the associated almost-complex structure, $J=K \circ\left(K^{*} K\right)^{-1 / 2}$.

We will be concerned with the eigenstates of low energy of the sequence of operators

$$
\square_{k}:=-\nabla^{(k)^{*}} \nabla^{(k)}-k \operatorname{Tr}^{+} K,
$$

where $\nabla^{(k)}$ is the connection on the $k$-th tensor power $L^{k} \rightarrow X$. The parameter $1 / k=\hbar$ plays the role of Planck's constant, and the operator $\frac{1}{k^{2}} \square_{k}$ is a perturbation

Received by the editors February 15, 2005.

2000 Mathematics Subject Classification. Primary 81Q20; Secondary 81S10.

The first author was supported in part by NSF grant DMS-0204985.

The second author was supported in part by NSF grant DMS-0070690. 
of a magnetic Schrödinger operator by a suitable potential term times $\hbar$. The precise meaning of "low-energy states" is the one implied by the following lemma.

Lemma 1.1 (10, 11]). There exist constants $\epsilon, M>0$ such that for large $k$ the spectrum of $\square_{k}$ is contained in

$$
(-\epsilon, \epsilon) \cup[M k, \infty) .
$$

A recent proof of this fact by X. Ma and G. Marinescu, [11, uses only the Lichnerowicz formula for the Spin-c Dirac operator associated with the almostKähler manifold $(X, \omega, J)$.

Let $\mathcal{H}_{k} \subset L^{2}\left(L^{k}\right)$ be the span of the low-lying eigensections of $\square_{k}$ identified by the lemma. The Kähler case corresponds precisely to $K=J$ and $J$ integrable. If in addition $L$ has a complex structure we can identify (noting that $\operatorname{Tr}^{+} K=n$ ) $-\nabla^{(k)^{*}} \nabla^{(k)}-n k=(1 / 4) \bar{\partial}^{*} \bar{\partial}$. Thus $\mathcal{H}_{k}$ would consist precisely of the holomorphic sections of $L^{k}$.

It is possible to start with a symplectic manifold $(X, \omega)$ and a Hermitian line bundle with connection $L \rightarrow X$ whose curvature is $\omega$. Then, by choosing a compatible almost-complex structure, $J$, one is led to the almost-Kähler case ( $K=J$ but not integrable), and the $\mathcal{H}_{k}$ are defined for $k$ sufficiently large. The almostKähler quantization scheme we proposed in [4] consists of taking $\mathcal{H}_{k}$ as the quantum Hilbert space corresponding to $\hbar=k^{-1}$. This is a direct analogue of the standard Kähler quantization.

Our main result is a precise description of the asymptotics, as $k \rightarrow \infty$, of the sequence of orthogonal projectors

$$
\Pi_{k}: L^{2}\left(L^{k}\right) \rightarrow \mathcal{H}_{k} .
$$

We will show that these projectors have the same structure as in the Kähler case. To state our results more precisely, we introduce the circle bundle $P \rightarrow X, P \subset L^{*}$. Then the sections of $L^{k} \rightarrow X$ are naturally identified with the space of functions on $P$ that transform under the circle action on $P$ by multiplication by the character $e^{i k \theta}$. Thus we can regard each $\mathcal{H}_{k}$ as a subspace of $L^{2}(P)$, and extend the orthogonal projection to $\Pi_{k}: L^{2}(P) \rightarrow \mathcal{H}_{k}$. The analogue of the Szegö projector from the Kähler case would then be the full projection $\Pi=\sum \Pi_{k}$. Our result is that $\Pi$ has the microlocal structure of the Szegö projector. A central role will be played by the submanifold $\mathcal{Z} \subset T^{*} P \backslash\{0\}$ of the punctured cotangent bundle of $P$ consisting of the positive multiples of the connection form. ( $\mathcal{Z}$ consists of the vertical covectors; one could also describe it as the contact ray for the contact structure on $P$ induced by the connection.) Our main result is the following.

Theorem 1.2. The projector $\Pi$ defined above is a Hermite FIO with Schwarz kernel in the class $I^{1 / 2}\left(P \times P, \mathcal{Z}^{\triangle}\right)$, where $\mathcal{Z}^{\triangle}$ is the diagonal relation $\{(\zeta, \zeta) ; \zeta \in$ $\mathcal{Z}\} \subset T^{*}(P \times P)$.

Results on semiclassical asymptotics for quantized Kähler manifolds have been derived in [1, 2, 3, 4, 5, using the structure of the Szegö projector as a Hermite FIO. Theorem 1.2 allows immediate extension of these results to the case of "almostKähler" quantization, i.e. quantization of a symplectic manifold with compatible almost structure using the spectral projector $\Pi$ defined above as an analogue of the Szegö projector. All of these are based on the same principle: the singularities of the Schwarz kernel $\Pi$ described in Theorem 1.2 correspond to the large $k$ behavior of the (finite-rank) projectors $\Pi_{k}$. 
To understand this connection, one can take a Fourier decomposition of the kernel of $\Pi$ as in Lemma 3.4 of [5]. The result is:

Corollary 1.3. As $k \rightarrow \infty$, the kernel of $\Pi_{k}$ has an asymptotic expansion (written w.r.t. some local coordinate system and trivialization of $L^{k}$ ) of the form

$$
\Pi_{k}(x, y) \sim \sum_{m \in \mathbb{N}_{0} / 2} k^{n-m} e^{i k \theta(x, y)} f_{m}(x, y, \sqrt{k}(x-y)),
$$

where the real phase $\theta(x, y)$ is determined by the relation $\mathcal{Z}^{\triangle}$ and $f_{m}(x, y, u)$ is rapidly decreasing as a function of $u \in \mathbb{R}^{2 n}$.

In the process of proving Theorem 1.2 we will also compute the symbol of $\Pi$ as a Hermite FIO, which is the invariant object associated to the leading term $f_{0}$ in the expansion above (see 33). The symbol is essentially identical to that of the Szegö projector in the Kähler case.

If we denote by $\psi_{j}^{(k)}, j=1, \ldots, d_{k}$, the (normalized) low energy eigenfunctions of $\square_{k}$ which span $\mathcal{H}_{k}$, then (1.5) gives an asymptotic expansion for the orthogonal projection onto these states. In particular, taking the symbol into account (see Theorem 4.1 of [5]) we have:

Corollary 1.4. The orthogonal projection onto $\mathcal{H}_{k}$ has an asymptotic expansion in $k$ with leading behavior

$$
\sum_{j=1}^{d_{k}} \psi_{j}^{(k)}(x) \overline{\psi_{j}^{(k)}(y)} \sim\left(\frac{k}{2 \pi}\right)^{n} e^{i k \theta(x, y)} e^{-\frac{k}{2} d(x, y)^{2}}+O\left(k^{n-1 / 2}\right) e^{-\frac{k}{2} d(x, y)^{2}},
$$

uniformly in $x, y$, where the linearization of the phase $\theta(x, y)$ at $y=x$ is the bilinear form associated to $K_{x}$ and $d(x, y)$ is the Riemannian distance.

These asymptotic results are completely analogous to results on the Szegö projector in the Kähler case; see [16. In the symplectic context, the existence of projectors on $L^{2}(P)$ with the microlocal structure of the Szegö projector (generalized Toeplitz structures) was established in the Appendix of [6]. That construction produces a zeroth-order pseudodifferential "correction" to $\square_{k}, R_{k}$, such that the orthogonal projection onto the (exact) kernel of $\square_{k}+R_{k}$ is a Szegö projector. In the process one loses any direct connection between $\mathcal{H}_{k}$ and eigenfunctions of $\Delta_{k}$. The main point of Theorem 1.2 is that we can perform this construction without the correction term, using the actual low-energy eigenfunctions. The results in Corollaries 1.3 and 1.4 would hold for any generalized Szegö projector, and Theorem 1.2 has allowed us to cast them as asymptotic spectral results for the Laplacian.

It is natural to ask whether the projector, $\Pi$, is a Fourier integral operator with complex phase (as in the Kähler case). Our method of proof, which we outline below, involves taking functions of a certain operator with symplectic characteristics, which results in general Hermite FIOs. It would be easy to deduce from our results the existence of a projector which is a complex FIO and whose principal symbol is as in the Kähler case, thereby re-proving the existence theorem of the appendix of 6. However, a priori that projector does not have to have the same range as $\Pi$.

Plan of the proof. The analysis is carried out naturally on the circle bundle, $P$. The connection form, the Riemannian metric on $X$, and the standard metric on $S^{1}$ combine to define a 'Kaluza-Klein' metric $P$ such that the projection $P \rightarrow X$ is a Riemannian submersion with totally geodesic fibers. (On horizontal subspaces 
the metric is defined by pullback from $X$, and horizontal and vertical subspaces are made orthogonal to each other.) Let $\Delta_{P}$ denote the Laplace-Beltrami operator on $P$ defined by this metric. The horizontal Laplacian, $\Delta_{H}$, is the operator defined by the identity

$$
\Delta_{P}=D_{\theta}^{2}+\Delta_{H} .
$$

Here $D_{\theta}=-i \partial / \partial \theta$ is differentiation with respect to the vector field generating the circle action on $P$. Since the circle action is by isometries, $\Delta_{P}$ commutes with $D_{\theta}$ and hence with $\Delta_{H}$. Under the natural identification of the sections of $L^{k}$ with the $k$-the eigenspace of $D_{\theta}$, the Laplacian $-\nabla^{(k)^{*}} \nabla^{(k)}$ corresponds to the restriction of $\Delta_{H}$ to that subspace. Therefore, under said correspondence, the operators $\left\{\square_{k}\right\}$ "roll up" to the operator

$$
\Delta_{H}-\left(\operatorname{Tr}^{+} K\right) D_{\theta},
$$

where we continue to denote by $\operatorname{Tr}^{+} K$ the pull-back of (1.3) to $P$. (Note that the associated multiplication operator commutes with $D_{\theta}$.) We will construct the projector, $\Pi$, by taking suitable functions of the first order operator

$$
\left(D_{\theta}\right)^{-1} \Delta_{H}-\operatorname{Tr}^{+} K \text {. }
$$

A complication is that this operator is a singular $\Psi \mathrm{DO}$ (because $D_{\theta}$ is not elliptic), but the singularities occur away from the characteristic variety of $\Delta_{H}$. We will therefore in fact work with an operator, $A$, which is microlocally equal to (1.7) away from the characteristic variety of $D_{\theta} . A$ is a standard $\Psi D O$ with double symplectic characteristics and such that the spectral projector of $A$ onto an interval $(-a, a)$ for small $a$ differs from $\Pi$ by an operator of finite rank.

The construction of the projector $\Pi$ is based on the following result.

Theorem 1.5. Let $A$ be an operator microlocally equal to the operator (1.7) in a conic neighborhood of the characteristics $\mathcal{Z}$, and let $\phi \in C_{0}^{\infty}(\mathbb{R})$. Then the operator

$$
\hat{\phi}(A):=\int_{\mathbb{R}} e^{-i t A} \phi(t) d t
$$

is a Hermite FIO with Schwarz kernel in the class $I^{1 / 2}\left(P \times P, \mathcal{Z}^{\triangle}\right)$.

We will identify the symbol of $\hat{\phi}(A)$ with an object that associates to each point of $\mathcal{Z}$ the following function of the harmonic oscillator on the symplectic normal bundle of $\mathcal{Z}$ :

$$
\hat{\phi}(0)|0\rangle\left\langle 0\left|+\sum_{\nu \neq 0} \hat{\phi}(2 \kappa \cdot \nu)\right| \nu\right\rangle\langle\nu| .
$$

Here the sum is over $\nu \in \mathbb{N}_{0}^{n}$ and $|\nu\rangle$ is the standard harmonic oscillator basis associated to the symplectic normal spaces.

With a suitable choice of test function, $\phi$, the operator $\hat{\phi}(A)$ is close to the spectral projector $\Pi$. A Neumann series argument finishes the construction of $\Pi$, proving Theorem 1.2. The construction shows furthermore that the symbol of $\Pi$ is projection onto the ground state of the harmonic oscillator on the symplectic normal bundle of $\mathcal{Z}$ :

$$
\sigma(\Pi)=|0\rangle\langle 0|,
$$

i.e. the the symbol is the same as for the Szegö projector in the Kähler case.

As we were finishing writing this paper Xiaonan Ma and George Marinescu forwarded us their preprint, 12, where they obtain, among other things, a description of the projectors $\Pi_{k}$ (by methods quite different from ours). 


\section{Hermite Fourier INTEGRAL OPERATORS}

A Hermite FIO differs from a standard FIO in having a canonical relation that is an isotropic, rather than Lagrangian, submanifold of the cotangent bundle. This generalization was motivated by the structure of the Szegö projector on the boundary of a strictly pseudoconvex domain.

For completeness, we begin by recalling the definition of a Hermite distribution. Let $M$ be a smooth manifold and $\Sigma \subset T^{*} M \backslash\{0\}$ a homogeneous isotropic submanifold.

Let $B$ be an open conic subset of $\left(\mathbb{R} \times \mathbb{R}^{n}\right) \backslash\{0\}$, given the coordinates $(\tau, \eta)$. A non-degenerate phase function is a function $\psi \in C^{\infty}(M \times B, \mathbb{R})$ which satisfies:

(1) $\psi(x, \tau, \eta)$ is homogeneous in $(\tau, \eta)$.

(2) $d \psi$ is nowhere zero.

(3) The critical set of $\psi$,

$$
C_{\psi}=\left\{(x, \tau, \eta) ;\left(d_{\tau} \psi\right)_{(x, \tau, \eta)}=\left(d_{\eta} \psi\right)_{(x, \tau, \eta)}=0\right\},
$$

intersects the the space $\eta_{1}=\ldots=\eta_{n}=0$ transversally.

(4) The map $(x, \tau, \eta) \mapsto\left(\frac{\partial \psi}{\partial \tau}, \frac{\partial \psi}{\partial \eta_{1}}, \ldots, \frac{\partial \psi}{\partial \eta_{n}}\right)$ has rank $n+1$ at every point of $C_{\psi}$, i.e. $\psi$ is non-degenerate.

Define the map $F: C_{\psi} \rightarrow T^{*} M$ by $(x, \tau, \eta) \mapsto\left(x,\left(d_{x} \psi\right)_{(x, \tau, \eta)}\right)$. The image under $F$ of the subspace $\{\eta=0\} \subset C_{\psi}$ is homogeneous isotropic submanifold of $T^{*} M$ of dimension $n+1$. If this image is equal to $\Sigma$, then we say that $\psi$ parametrizes $\Sigma$.

Definition 2.1. The space $I^{m}(M, \Sigma)$ of Hermite distributions consists of distributions on $M$ which have a local representation as oscillatory integrals of the form

$$
\int e^{i \psi(x, \tau, \eta)} a\left(x, \tau, \frac{\eta}{\sqrt{\tau}}\right) d \tau d \eta
$$

where $\psi$ parametrizes $\Sigma$, and the amplitude $a(x, \tau, u)$ has the following properties (see $\S 3$ of [6] for the precise formulation of the estimates):

(1) $a(x, \tau, u)$ is rapidly decreasing as a function of $u$.

(2) $a(x, \tau, u)$ is cutoff to be zero near $\tau=0$.

(3) For sufficiently large $\tau, a(x, \tau, u)$ admits an expansion of the form

$$
a(x, \tau, u) \sim \sum_{i=0}^{\infty} \tau^{m_{i}} a_{i}(x, u),
$$

where each $m_{i}$ is either integer or half-integer, with $m_{0}=m-1 / 2$ and $m_{i} \rightarrow-\infty$.

The conditions for a phase function to parametrize an isotropic submanifold ensure that a distribution in $I^{m}(M, \Sigma)$ will have wave-front set contained in $\Sigma$.

2.1. The operator $A$. We will now construct the operator $A$ that suitably approximates the operator (1.7). The idea is to construct a suitable parametrix of $D_{\theta}$ that we will denote by $Q$.

We start with the rolled-up version of the $\left\{\square_{k}\right\}$, namely

$$
S=\Delta_{H}-\left(\operatorname{Tr}^{+} K\right) D_{\theta} .
$$

Although $S$ is not elliptic, the operator $\Delta_{P}-\left(\operatorname{Tr}^{+} K\right) D_{\theta}=S+D_{\theta}^{2}$ is, and it commutes with $S$. We can therefore find an orthonormal basis of $L^{2}(P)$ of joint 
eigenfunctions of $S$ and $D_{\theta}$ :

$$
S\left(\psi_{j}^{k}\right)=\lambda_{j}^{k} \psi_{j}^{k}, \quad D_{\theta}\left(\psi_{j}^{k}\right)=k \psi_{j}^{k}, \quad \lambda_{1}^{k} \leq \lambda_{2}^{k} \leq \cdots .
$$

Note that, for each $k$, the $\lambda_{j}^{k}, j=1,2, \ldots$, are the eigenvalues of $\square_{k}$. Therefore, the spectral drift phenomenon of Lemma 1.1 has the following interpretation in terms of the $\lambda$ 's: Denote the dimension of $\mathcal{H}_{k}$ by $d_{k}$. Then, for all sufficiently large $k$

$$
-\epsilon<\lambda_{1}^{k} \leq \cdots \leq \lambda_{d_{k}}^{k}<\epsilon \text { and } M k \leq \lambda_{d_{k}+1}^{k} \leq \lambda_{d_{k}+2}^{k} \leq \cdots .
$$

Since the $\lambda_{j}^{k}+k^{2}$ are the eigenvalues of an elliptic operator with positive symbol, only finitely many of them can be negative. We can therefore form an operator that we will denote by

$$
F:=\sqrt{S+D_{\theta}^{2}}
$$

because we can arrange that $F$ be a function of $S+D_{\theta}^{2}$ and be such that $F^{2}-\left(S+D_{\theta}^{2}\right)$ is finite rank. The operator $F$ is a standard elliptic first-order $\Psi$ DO commuting with $S$ and with $D_{\theta}$.

Lemma 2.2. There exist non-negative cut-off functions, $f, g \in C^{\infty}(\mathbb{R})$, such that the operator

$$
Q:=f\left(D_{\theta}^{2} F^{-2}\right) D_{\theta}^{-1}+g\left(S F^{-2}\right) F^{-1}
$$

has the following properties:

(1) $Q$ is a classical elliptic $\Psi D O$ of order $(-1)$.

(2) In a conic neighborhood of the submanifold $\mathcal{Z} \subset T^{*} P \backslash\{0\}$, the symbol of $Q$ is $\sigma\left(D_{\theta}\right)^{-1}$.

(3) If $q_{j}^{k}$ denote the eigenvalues of $Q$ (so that $Q\left(\psi_{j}^{k}\right)=q_{j}^{k} \psi_{j}^{k}$ ), then there exists $c>0$ such that for all $k$ sufficiently large:

(a) $q_{j}^{k}=k^{-1}$ for all $j=1,2, \ldots, d_{k}$.

(b) For all $j>d_{k}, q_{j}^{k} \geq c / k$.

Proof. We begin by noting that the arguments of $f$ and $g$ above are classical zerothorder $\Psi$ DOs, and therefore for any choice of smooth functions $f$ and $g$ the operators $f\left(D_{\theta}^{2} F^{-2}\right)$ and $g\left(S F^{-2}\right)$ are classical $\Psi$ DOs of order zero. If we choose $f(x)$ identically equal to zero near $x=0$, then $f\left(D_{\theta}^{2} F^{-2}\right) D_{\theta}^{-1}$ is a classical $\Psi$ DO of order $(-1)$, which implies (1). Moreover, if we choose $f(x)$ identically equal to one near $x=1$, then condition (2) above is satisfied as well.

For any $f$ and $g$ the eigenvalues of $Q$ can be written as

$$
q=k^{-1} f\left(\frac{k^{2}}{\lambda+k^{2}}\right)+\frac{1}{\sqrt{\lambda+k^{2}}} g\left(\frac{\lambda}{\lambda+k^{2}}\right),
$$

where we have written $\lambda$ for $\lambda_{j}^{k}$, for simplicity. Note that the arguments of $f$ and $g$ above add up to one. Since $f(x)$ is being chosen identically equal to one near $x=1, f\left(\frac{k^{2}}{\lambda+k^{2}}\right)=1$ if $k$ is large and $\lambda$ bounded. If we choose $g(x)$ to be zero near $x=0$, this, together with the previous choices, implies condition (3)(a). It remains to be shown that, in addition, one can choose $f$ and $g$ so as to ensure condition (3) (b). 
If $j>d_{k}$, then $\lambda_{j}^{k}>M k$. We distinguish two sub-regimes of this case. First, if $M k<\lambda \leq a k^{2}$ for some $a>0$, then

$$
\frac{k^{2}}{\lambda+k^{2}} \geq a / 2
$$

and so we can arrange for $f\left(\frac{k^{2}}{\lambda+k^{2}}\right)$ to be bounded below. On the other hand, if $\lambda>a k^{2}$, then $\frac{\lambda}{\lambda+k^{2}}>a$. Therefore, if $f(x)<1 / 10$ (for example) implies $g(1-x)=1$, then $q$ is bounded below by a constant times $1 / k$ as well.

We now define the operator $A$ in terms of the operator $Q$ by

$$
A:=Q \circ S=Q \circ\left(\Delta_{H}-\left(\operatorname{Tr}^{+} K\right) D_{\theta}\right) .
$$

Its main properties are summarized by the following.

\section{Corollary 2.3.}

(1) A is a first-order classical $\Psi D O$ with double characteristics, $\mathcal{Z}$. The symbol of $A$ is identical to $\sigma\left(\Delta_{H}\right) / \sigma\left(D_{\theta}\right)$ in a conic neighborhood of $\mathcal{Z}$.

(2) If $\alpha_{j}^{k}$ denote the eigenvalues of $A$ (so that $A\left(\psi_{j}^{k}\right)=\alpha_{j}^{k} \psi_{j}^{k}$ ), then there exists $C>0$ such that for all $k$ sufficiently large:

(a) $\alpha_{j}^{k}=\frac{1}{k} \lambda_{j}^{k} \in(-\epsilon / k, \epsilon / k)$ for all $j=1,2, \ldots, d_{k}$.

(b) For all $j>d_{k}, \alpha_{j}^{k} \geq C$.

Since $A$ is a function of $S$, we can characterize the $\mathcal{H}_{k}$ as the span of the joint eigenfunctions of $A$ and $D_{\theta}$ whose eigenvalues are $O(1 / k)$ from zero, with $A$ firstorder.

2.2. Proof of Theorem 1.5. The family of operators $\left\{e^{-i t A}\right\}_{t}$ is a smooth family of Fourier integral operators, [15, Ch. VIII, $\S 8$. The Schwartz kernel of the family, $U(t, x, y)$, is a distribution on $\mathbb{R} \times P \times P$ with a wave-front set equal to

$$
\Gamma:=\left\{(t, \tau ; x, \xi ; y,-\eta) ; \tau=p(x, \xi) \text { and }(y, \eta)=f_{t}(x, \xi)\right\},
$$

where $p$ is the principal symbol of $A$ and $f_{t}$ is the Hamiltonian flow of this symbol. To determine the wave-front set of $\hat{\phi}(A)$, note that the operator from $C^{\infty}(\mathbb{R} \times P \times P)$ to $C^{\infty}(P \times P)$ given by

$$
v(t, x, y) \mapsto \int_{\mathbb{R}} v(t, x, y) \phi(t) d t
$$

is a Fourier integral operator with canonical relation

$$
\mathcal{C}:=\{(t, \tau=0 ; x, \xi ; y, \eta ; x, \xi ; y, \eta)\} .
$$

Applying this operator to the distribution $U(t, x, y)$ gives $\hat{\phi}(A)$, whose wave front set is thereby seen to be contained in $\mathcal{C} \circ \Gamma=\mathcal{Z}^{\triangle}$.

Therefore, by the calculus of wave-front sets, to compute the Schwartz kernel of $\hat{\phi}(A)$ modulo smooth functions we can microlocalize $U(t, x, y)$ to any neighborhood of the set $\{(t, \tau ; x, \xi ; y,-\eta) ; \tau=0\}$. Let $\mathcal{V}$ be a conic neighborhood of this set, specifically,

$$
\mathcal{V}=\left\{(t, \tau ; x, \xi ; y,-\eta) ;|\tau|<\delta \sqrt{\|\xi\|^{2}+\|\eta\|^{2}}\right\}
$$

where $\|\xi\|$ is the norm with respect to the Kaluza-Klein metric on $P$. Note that (with a different constant, $\delta$ )

$$
\Gamma_{\delta}:=\mathcal{V} \cap \Gamma=\left\{(t, \tau ; x, \xi ; y,-\eta) ; \tau=p(x, \xi)<\delta\|\xi\| \text { and }(y, \eta)=f_{t}(x, \xi)\right\}
$$


Lemma 2.4. Let $W \subset P \times P$ be a neighborhood of the diagonal, and let $\epsilon>0$. Then there exists a small enough $\delta>0$ such that the projection

$$
\begin{array}{ccc}
\Gamma_{\delta} & \rightarrow & \mathbb{R} \times P \times P \\
(t, \tau ; x, \xi ; y,-\eta) & \mapsto & (t, x, y)
\end{array}
$$

takes its values in $[-\pi-\epsilon, \pi+\epsilon] \times W$.

Proof. Let $\kappa$ denote the symbol of $D_{\theta}$, and let

$$
\|\cdot\|^{2}=\kappa^{2}+h(\cdot)^{2}
$$

(that is, $h$ denotes the norm of the horizontal component of a covector). Then, $p=h^{2} / \kappa$, and therefore

$$
p<\delta\|\cdot\| \quad \Leftrightarrow \quad h^{2}<\delta \kappa \sqrt{h^{2}+\kappa^{2}} .
$$

The flow $\left\{f_{t}\right\}$ is homogeneous of degree zero, therefore, to analyze its behavior we can restrict our attention to the set $\{\kappa=1\}$. But

$$
(\kappa=1 \text { and } p<\delta\|\cdot\|) \Rightarrow\left(\kappa=1 \text { and } h^{2}<\delta(h+1)\right),
$$

and $h^{2}<\delta(h+1)$ implies that $h=o(\delta)$. Therefore, if $(t, \tau ; x, \xi ; y,-\eta) \in \Gamma_{\delta} \cap\{\kappa=$ $1\}$, then $h(x, \xi)=o(\delta)$, and in particular $(x, \xi)$ is close to $\Sigma \cap\{\kappa=1\}$. Now note that both $h$ and its Hamilton vector field vanish on $\Sigma$. Therefore, if $\delta$ is small enough, the trajectory of $(x, \xi)$ by $\left\{f_{t}\right\}$ remains close to $(x, \xi)$ even for times that are of the order of $\pi$.

To prove that $\hat{\phi}(A)$ is a Hermite operator, recall (see [14) that one can write the Schwartz kernel of $e^{-i t A}$ as an oscillatory integral with Hörmander's phase function, i.e. of the form

$$
U(t, x, y)=\int e^{i[\psi(t, x, y, \eta)-t p(y, \eta)]} a(t, x, y, \eta) d \eta,
$$

where $p$ is the principal symbol of $P$ and $\psi$ is homogeneous of degree one in $\eta$ and satisfies

$$
\begin{gathered}
p\left(x, d_{x} \psi(x, y, \eta)\right)=p(y, \eta), \\
\psi(x, y, \eta)=0 \quad \text { if }\langle x-y, \eta\rangle=0, \\
d_{x} \psi(x, y, \eta)=\eta \quad \text { if } x=y .
\end{gathered}
$$

The phase function $\psi$ parametrizes the conormal to the diagonal on $P \times P$, and therefore the variable $\eta$ corresponds with a covector at $y$. The amplitude $a(t, x, y, \eta)$ is of the form

$$
a(t, x, y, \eta) \sim \sum_{j=0}^{\infty} a_{j}(t, x, y, \eta),
$$

where $a_{j}$ is positive homogeneous in $\eta$ of order $(-j)$. These amplitudes must satisfy the transport equations which is always possible for $t$ sufficiently small.

Let us write the symbol $p$, in our case. Given a covector $\eta$, write it as $\eta=\left(\eta_{H}, \kappa\right)$ with $\eta_{H}$ horizontal and $\kappa$ a variable dual to $\partial_{\theta}$. Then

$$
p(y, \eta)=\frac{\left\|\eta_{H}\right\|^{2}}{\kappa} \text {. }
$$


Multiplying by $\phi(t)$ and integrating with respect to $t$ we obtain that the Schwartz kernel of $A_{\phi}$ is given by oscillatory integrals of the form

$$
\int e^{i \psi(x, y, \eta)} f\left(x, y, \eta, \frac{\left\|\eta_{H}\right\|^{2}}{\kappa}\right) d \eta d \kappa,
$$

where

$$
f(x, y, \eta, \kappa)=\int e^{-i t \kappa} \phi(t) a(t, x, y, \eta) d t .
$$

Since $\phi$ is compactly supported, $f$ is rapidly decreasing in the $\kappa$ variable. Thus, given the polyhomogeneous expansion of $a(t, x, y, \eta)$, we see that (2.6) is the local form of an element of $I^{0}\left(P \times P, \mathcal{Z}^{\triangle}\right)$. (Technically $\psi$ parametrizes the conormal to the diagonal, rather than $\mathcal{Z}^{\triangle}$. But because of the rapid decay in the $\eta_{H}$ variables, specializing to a phase function parametrizing $\mathcal{Z}^{\triangle}$ would introduce only smooth error terms.)

\section{The symbol COMPUTATION}

The symbol of a Hermite Fourier distribution in $I^{l}(M, \Sigma)$ is a symplectic spinor, which is a half-density along $\Sigma$ tensored with a smooth vector in the metaplectic representation associated to the symplectic normal to $\Sigma$. Denoting $\Sigma_{\rho}:=T_{\rho} \Sigma$, we can write the space of symplectic spinors at $\rho$ as

$$
\operatorname{Spin}\left(\Sigma_{\rho}\right)=\bigwedge^{1 / 2}\left(\Sigma_{\rho}\right) \otimes H_{\infty}\left(\Sigma_{\rho}^{\circ} / \Sigma_{\rho}\right) .
$$

Here we use $\Sigma_{\rho}^{\circ}$ to denote the symplectic orthogonal to $\Sigma_{\rho}$, i.e. $\Sigma_{\rho}^{\circ}=\left\{w \in T_{\rho}^{*} P\right.$ : $\left.\omega(w, v)=0 \forall v \in \Sigma_{\rho}\right\}$. The symplectic normal, $\Sigma_{\rho}^{\circ} / \Sigma_{\rho}$, is thus a symplectic subspace. For any symplectic vector space we have a metaplectic representation, and we use $H_{\infty}$ to denote the set of smooth vectors within that representation. In reference to Definition 2.1, the half-density encodes the usual dependence of the leading amplitude $a_{0}(x, \tau, u)$ on local coordinates, whereas the smooth vector is the invariant form of the rapidly decreasing function of $u$. For more complete definitions as well as the essential theorems underlying the calculation in this section, we must refer the reader to [6, 9 .

From Theorem 1.5 we know that $\hat{\phi}(A) \in I^{0}\left(P \times P, \mathcal{Z}^{\triangle}\right)$. Let $\phi \in \mathcal{Z}$ and denote the tangent spaces

$$
V_{\rho}=T_{\rho}\left(T^{*} P\right), \quad Z_{\rho}:=T_{\rho} \mathcal{Z},
$$

so that $Z_{\rho} \subset V_{\rho}$ is a symplectic subspace. The tangent space of $T^{*}(P \times P)$ is $W_{\rho}:=$ $V_{\rho} \times V_{\rho}$, where the second factor carries the opposite symplectic form (following the usual convention for canonical relations of FIO's), and $Z_{\rho}^{\triangle} \subset W_{\rho}$ is thus isotropic. With this setup it is a simple exercise to work out that

$$
\left(Z_{\rho}^{\triangle}\right)^{\circ} /\left(Z_{\rho}^{\triangle}\right) \cong Z_{\rho}^{\circ} \oplus Z_{\rho}^{\circ} .
$$

The smooth vector part of the symbol of $\hat{\phi}(A)$ is thus an element of $H_{\infty}\left(Z_{\rho}^{\circ}\right) \otimes$ $H_{\infty}\left(Z_{\rho}^{\circ}\right)$. By the identification $Z_{\rho}^{\triangle} \cong Z_{\rho}$ and the symplectic structure on the latter, $Z_{\rho}^{\triangle}$ carries a natural half-form. Thus we have a canonical identification

$$
\operatorname{Spin}\left(Z_{\rho}^{\triangle}\right) \cong \operatorname{End}\left(H_{\infty}\left(Z_{\rho}^{\circ}\right)\right) \text {. }
$$

One last observation is needed to state the main result. The metaplectic group of $Z_{\rho}^{\circ}$ acts on $H_{\infty}\left(Z_{\rho}^{\circ}\right)$, by definition. Thus an element of the symplectic algebra

of $Z_{\rho}^{\circ}$ acts on $H_{\infty}\left(Z_{\rho}^{\circ}\right)$ via the infinitesimal representation $d \tau$. (We will describe 
this action in more detail below.) Now the projection $T_{\rho}\left(T^{*} P\right) \rightarrow T_{\pi(\rho)} P$ naturally identifies $Z_{\rho}^{\circ}$ with $T_{\pi(\rho)} X$. Hence the bundle automorphism $K$ defined by (1.2) can be lifted to an element of the symplectic algebra of $Z_{\rho}^{\circ}$. Under a canonical identification $Z_{\rho}^{\circ} \cong \mathbb{R}_{(x, \eta)}^{2 n}$ such that $H_{\infty}\left(Z_{\rho}^{\circ}\right) \cong \mathcal{S}\left(\mathbb{R}_{\eta}^{n}\right)$ and

$$
K=\left(\begin{array}{cc}
0 & (\kappa) \\
(-\kappa) & 0
\end{array}\right)
$$

(where $(\kappa)$ denotes the $n \times n$ diagonal matrix with entries $\kappa_{1}, \ldots, \kappa_{n}$ ), we have

$$
d \tau(K)=-\sum_{j=1}^{n} \kappa_{j}\left(\frac{\partial^{2}}{\partial \eta_{j}^{2}}+\eta_{j}^{2}\right)
$$

i.e. the harmonic oscillator Hamiltonian. For this operator we have the standard orthonormal basis of eigenfunctions $|\nu\rangle \in H_{\infty}\left(Z_{\rho}^{\circ}\right), \nu=\left(\nu_{1}, \ldots, \nu_{n}\right)$, such that

$$
d \tau(K)|\nu\rangle=\left(\kappa \cdot \nu+\operatorname{Tr}^{+} K / 2\right)|\nu\rangle,
$$

where $\kappa \cdot \nu=\sum_{j} \kappa_{j} \nu_{j}$.

Proposition 3.1. Under the identification (3.1),

$$
\sigma(\hat{\phi}(A))=\sum_{\nu} \hat{\phi}(2 \kappa \cdot \nu)|\nu\rangle\langle\nu| .
$$

3.1. The transport equation. To prove Proposition 3.1, we will use test distributions in the class $I^{l}(P, \mathcal{R})$, associated to an isotropic ray $\mathcal{R}=\left\{\left(p, r \alpha_{p}\right) ; r>0\right\} \subset \mathcal{Z}$, for some fixed $p \in P$. It suffices to make our calculation in the tangent space to $T^{*} P$ at the point $\rho=\left(p, \alpha_{p}\right)$ (because symbols will be homogeneous in the ray coordinate). Let $R_{\rho}=T_{\rho} \mathcal{R}$, which is an isotropic subspace of $Z_{\rho}$. We can identify the symplectic normal space

$$
R_{\rho}^{\circ} / R_{\rho} \cong E_{\rho} \oplus Z_{\rho}^{\circ}
$$

where $E_{\rho}$ is the symplectic normal of $R_{\rho}$ as a subspace of $Z_{\rho}$. Thus

$$
\operatorname{Spin}\left(R_{\rho}\right) \cong \bigwedge^{1 / 2}\left(R_{\rho}\right) \otimes H_{\infty}\left(E_{\rho}\right) \otimes H_{\infty}\left(Z_{\rho}^{\circ}\right) .
$$

Consider the action of the operator $A$ on $I^{l}(P, \mathcal{R})$. From [9], Theorem V, we obtain the following transport equation.

Proposition 3.2. Since $\sigma(A)$ vanishes to second order on $\mathcal{R}$, given $u \in I^{l}(P, \mathcal{R})$ we have $A u \in I^{l}(P, \mathcal{R})$ and

$$
\sigma(A u)=d \tau(\operatorname{Hess}(\sigma(A))) \cdot \sigma(u)+\sigma_{\text {sub }}(A) \sigma(u),
$$

where the Hessian of the symbol, Hess $(\sigma(A))$, acts on the smooth vector component of $\sigma\left(u_{t}\right)$ via the infinitesimal metaplectic representation $d \tau$.

To describe the symbol of $\sigma(A u)$ more explicitly, we introduce convenient local coordinates $(x, \theta ; \eta, \tau)$ for $T^{*} P$ such that $\rho=(0,0 ; 0,1)$. Here $x$ is assumed to be a geodesic normal coordinate for $X$, so that, if $\beta$ denotes the metric on $X$, then $\beta_{i j}=\delta_{i j}+O\left(x^{2}\right)$. The connection form is given by

$$
\alpha=d \theta+\sum \alpha_{j}(x) d x_{j},
$$

and we may assume, without loss of generality, that $\alpha_{j}(0)=0$ and $\partial_{i} \alpha_{j}(0)=\frac{1}{2} K_{i j}$, where $K_{i j}$ is the matrix of $K$ in these coordinates (which is the same matrix as $\omega$ since $g$ is normal at 0 ). 
The Kaluza-Klein metric on $P$, in block matrix form, is given by

$$
g=\left(\begin{array}{cc}
1 & \alpha^{t} \\
\alpha & \beta+\alpha \alpha^{t}
\end{array}\right)
$$

(considering $\alpha_{j}$ to be a column vector) so that

$$
g^{-1}=\left(\begin{array}{cc}
1+\alpha^{t} \beta^{-1} \alpha & -\alpha^{t} \beta^{-1} \\
-\beta^{-1} \alpha & \beta^{-1}
\end{array}\right)
$$

From the connection between the symbol of $A$ and the symbol of $\Delta_{H}=\Delta_{P}-D_{\theta}^{2}$ in Corollary 2.3 and the form of $g^{-1}$ we see that

$$
\sigma(A)(x, \theta ; \eta, \tau)=\frac{1}{\tau}(\eta-\tau \alpha)^{t} \beta^{-1}(\eta-\tau \alpha) .
$$

The subprincipal symbol of $\Delta_{H}$ being zero, we also have $\sigma_{\text {sub }}(A)=-\operatorname{Tr}^{+} K$. We need the Hessian of $\sigma(A)$, which is

$$
\begin{aligned}
& \left.\frac{\partial^{2}}{\partial x_{i} \partial x_{j}} \sigma(A)\right|_{\rho}=2 \sum \partial_{i} \alpha_{k}(0) \partial_{j} \alpha_{k}(0)=-\frac{1}{2}\left(K^{2}\right)_{i j}, \\
& \left.\frac{\partial^{2}}{\partial x_{i} \partial \eta_{j}} \sigma(A)\right|_{\rho}=-2 \partial_{i} \alpha_{j}(0)=K_{i j}, \\
& \left.\frac{\partial^{2}}{\partial \eta_{i} \partial \eta_{j}} \sigma(A)\right|_{\rho}=2 \delta_{i j} .
\end{aligned}
$$

Thus, as an element of the symplectic algebra of $R_{\rho}^{\circ} / R_{\rho}$,

$$
\operatorname{Hess}(\sigma(A))=\left(\begin{array}{cc}
K & 2 I \\
\frac{1}{2} K^{2} & K
\end{array}\right)
$$

In order to express the transport equation in terms of the decomposition (3.2), we need to express Hess $(\sigma(A))$ as an element of the symplectic algebra of $E_{\rho} \oplus Z_{\rho}^{\circ}$. If we introduce coordinates

$$
\begin{aligned}
R_{\rho}^{\circ} / R_{\rho} & =\{(v, 0 ; \xi, 0)\}, \\
E_{\rho} & =\left\{\left(v_{1}, 0 ;-\left(K \cdot v_{1}\right) / 2,0\right)\right\}, \\
Z_{\rho}^{\circ} & =\left\{\left(v_{2}, 0 ;\left(K \cdot v_{2}\right) / 2,0\right)\right\},
\end{aligned}
$$

then the canonical transformation $T: E_{\rho} \oplus Z_{\rho}^{\circ} \rightarrow R_{\rho}^{\circ} / R_{\rho}$ is can be read off immediately:

$$
\begin{aligned}
& v=v_{1}+v_{2}, \\
& \xi=-\left(K \cdot v_{1}\right) / 2+\left(K \cdot v_{2}\right) / 2 .
\end{aligned}
$$

Conjugating the Hessian gives

$$
\begin{aligned}
T^{-1} \circ \operatorname{Hess}(\sigma(A)) \circ T & =\left(\begin{array}{cc}
1 / 2 & -K^{-1} \\
1 / 2 & K^{-1}
\end{array}\right)\left(\begin{array}{cc}
K & 2 \\
K^{2} / 2 & K
\end{array}\right)\left(\begin{array}{cc}
1 & 1 \\
-K / 2 & K / 2
\end{array}\right) \\
& =\left(\begin{array}{cc}
0 & 0 \\
0 & 2 K
\end{array}\right) .
\end{aligned}
$$

Thus, by Proposition 3.2, $\sigma(A u)$ is given in terms of the decomposition (3.2) by the operator $2 d \tau(K)-\operatorname{Tr}^{+} K$ acting on the $H_{\infty}\left(Z_{\rho}^{\circ}\right)$ component of $\sigma(u)$. If we take $u \in I^{l}(P, \mathcal{R})$ with symbol

$$
\sigma(u)=\mu \otimes a \otimes|\nu\rangle \in \bigwedge^{1 / 2}\left(R_{\rho}\right) \otimes H_{\infty}\left(E_{\rho}\right) \otimes H_{\infty}\left(Z_{\rho}^{\circ}\right),
$$


where $\{|\nu\rangle\}$ is the harmonic oscillator basis for $d \tau(K)$ introduced above, then the transport equation reduces to

$$
\sigma(A u)=(2 \kappa \cdot \nu) \sigma(u)
$$

3.2. Proof of Proposition 3.1. With the transport equation understood, the proof of Proposition 3.1 is fairly straightforward. Suppose $u \in I^{l}(P, \mathcal{R})$ with symbol as in (3.5), and let

$$
u_{t}=e^{-i t A} u
$$

Then $D_{t} u_{t}=-A u_{t}$, and hence, by the transport equation worked out above,

$$
D_{t} \sigma\left(u_{t}\right)=-2 \kappa \cdot \nu \sigma\left(u_{t}\right)
$$

It is thus clear that

$$
\sigma\left(u_{t}\right)=e^{-2 i t \kappa \cdot \nu} \sigma(u)
$$

whence

$$
\sigma(\hat{\phi}(A) u)=\hat{\phi}(2 \kappa \cdot \nu) \sigma(u)
$$

The result then follows from the interpretation of the smooth vector part of $\sigma(\hat{\phi}(A))$ as an endomorphism in (3.1).

\section{Constructing the projector}

To make the connection between $\hat{\phi}(A)$ and $\Pi$ we first choose $\phi \in C_{0}^{\infty}$ with the following properties:

(1) $\hat{\phi}(0)=1$ and for all integers $\ell \neq 0 \hat{\phi}(\ell)=0$.

(2) $\hat{\phi}(\xi)<1 / 2$ for all $\xi>\epsilon$, where $\epsilon$ is the constant of Lemma 1.1 .

Such functions exist; for example we can take $\phi$ to be the convolution of the characteristic function of $[-1,1]$ and a suitable function in $C_{0}^{\infty}$. A consequence of the first condition is that the symbol of $\hat{\phi}(A)$ is, at each point in $\Sigma$, the rank-one projector onto the ground state of the corresponding harmonic oscillator. Thus $\hat{\phi}(A)$ is a projector at the symbolic level.

We will now use a more or less well-known Neumann series argument to obtain the desired projector (analogously as in, e. g., 7]). The basic idea is to use the identity

$$
\frac{x-1 / 2}{\sqrt{1+4\left(x^{2}-x\right)}}+1 / 2=\Theta(x-1 / 2)
$$

where $\Theta$ is the Heaviside function. Note that, by the second property of $\phi$ above, $\Theta\left(\hat{\phi}(A)-\frac{1}{2} I\right)$ is the desired projector. Therefore we want to replace $x$ by $\hat{\phi}(A)$ in (4.1).

We begin with $E=4\left(\hat{\phi}(A)^{2}-\hat{\phi}(A)\right)$. Since the symbol of $\hat{\phi}(A)$ is a projector, then $E$ is a Hermite FIO of order $(-1 / 2)$, and therefore compact. Let us now define $(I+E)^{-1 / 2}$ by a Neumann series. Following the remarks above, it is then a simple matter to check that

$$
\Pi:=\left(\hat{\phi}(A)-\frac{1}{2} I\right)(I+E)^{-1 / 2}+\frac{1}{2} I
$$

is the desired projector. It remains to show that $\Pi$ is Hermite. The issue here is that there is no guarantee that the limit of the Neumann series for $(I+E)^{-1 / 2}$ produces a Hermite FIO. However, all partial sums of the series are Hermite. Let 
$(1+x)^{-1 / 2}=\sum_{l=0}^{\infty} c_{l} x^{l}$. Then, by a standard Borel summation argument, one can construct a Hermite FIO, $B \in I^{0}(P \times P, \mathcal{Z})$, such that for each $k$

$$
B-\sum_{l=0}^{k} c_{l} E^{l} \in I^{-\frac{k+1}{2}}(P \times P, \mathcal{Z}) .
$$

Let us now set

$$
R=\Pi-\left(\hat{\phi}(A)-\frac{1}{2}\right) B-\frac{1}{2} I .
$$

We will now show that $R$ is smoothing, which will imply that $\Pi$ is a Hermite FIO. A calculation shows that

$$
R=\left(\hat{\phi}(A)-\frac{1}{2} I\right)\left[\left(\sum_{l=0}^{\infty} c_{l} E^{l}\right)-B\right] .
$$

For each positive integer $k$, let us write the operator in brackets in the form

$$
\sum_{l=0}^{\infty} c_{l} E^{l}-B=\left(\sum_{l=0}^{k} c_{l} E^{l}-B\right)+E^{k+1} \sum_{l=0}^{\infty} c_{l+k+1} E^{l}
$$

and note that both terms map $H^{0} \rightarrow H^{(k+1) / 2}$ (the series above being bounded in $L^{2}$ ). Since this holds for any $k, R$ is smoothing and $\Pi$ is Hermite. Moreover, by construction,

$$
\sigma(\Pi)=\sigma(\hat{\phi}(A))=|0\rangle\langle 0| .
$$

\section{ACKNOWLEDGMENTS}

We wish to thank Victor Guillemin and Yves Colin de Verdière for helpful conversations.

\section{REFERENCES}

[1] M. Bordemann, E. Meinrenken, M. Schlichenmaier, Toeplitz quantization of Kähler manifolds and $g l(N), N \rightarrow \infty$ limits, Comm. Math. Phys. 165 (1994), 281-296. MR.1301849 (96f:58067)

[2] D. Borthwick, T. Paul and A. Uribe, Legendrian distributions with applications to relative Poincaré series, Invent. Math. 122 (1995), 359-402. MR1358981(97a:58188)

[3] D. Borthwick, T. Paul and A. Uribe, Semi-classical spectral estimates for Toeplitz operators, Anal. Inst. Fourier 48 (1998), 1189-1229. MR1656013 (2000c:58048)

[4] D. Borthwick and A. Uribe, Almost-Complex Structures and Geometric Quantization, Math. Res. Lett. 3 (1996), 845-861. MR 1426541 (98e:58084)

[5] D. Borthwick and A. Uribe, Nearly Kählerian embeddings of symplectic manifolds, Asian J. Math. 4 (2000), 599-620. MR1796696 (2001m:53166)

[6] L. Boutet de Monvel and V. Guillemin. The spectral theory of Toeplitz operators, Annals of Mathematics Studies No. 99, Princeton University Press, Princeton, N.J., 1981. MR0620794 (85j:58141)

[7] L. Boutet de Monvel and J. Sjöstrand, Sur la singularité des noyaux de Bergmann et de Szegö, Asterisque 34-35 (1976), 123-164. MR0590106 (58:28684)

[8] S. Donaldson, Symplectic submanifolds and almost complex geometry, J. Diff. Geom. 44 (1996), 666-705. MR:1438190 (98h:53045)

[9] V. Guillemin. Symplectic spinors and partial differential equations. Coll. Inst. CNRS n. 237, Géométrie Symplectique et Physique Mathématique, 217-252. MR0461591 (57:1576)

[10] V. Guillemin and A. Uribe, The Laplace operator on the $n$-th tensor power of a line bundle: eigenvalues which are uniformly bounded in n, Asymptotic Analysis 1 (1988), 105-113. MR0950009 (90a:58180)

[11] X. Ma and G. Marinescu, The spin-c Dirac operator on high tensor powers of a line bundle. Math. Zeitschrift 240 (2002), 651-664. MR.1924025 (2003h:58043) 
[12] X. Ma and G. Marinescu, Generalized Bergman kernels on symplectic manifolds, C. R. Math. Acad. Sci. Paris 339 (2004), 493-498. MR2099548 (2005k:58065)

[13] B. Shiffman and S. Zelditch, Asymptotics of almost holomorphic sections of ample line bundles on symplectic manifolds. J. Reine Angew. Math. 544 (2002), 181-222. MR1887895 (2002m:58043)

[14] M. Shubin, Pseudodifferential Operators and Spectral Theory, Springer, 1987. MR0883081 (88c:47105)

[15] F. Trèves, Introduction to pseudodifferential and Fourier integral operators, Vol. 2. Plenum Press, N.Y., 1982. MR0597145 (82i:58068)

[16] S. Zelditch, Szegö kernels and a theorem of Tian, Internat. Math. Res. Notices 6 (1998), 317-331. MR.1616718 (99g:32055)

Department of Mathematics and Computer Science, Emory University, Atlanta, Georgia 30322

E-mail address: davidb@math.emory.edu

Department of Mathematics, University of Michigan, Ann Arbor, Michigan 48109

E-mail address: uribe@math.1sa.umich.edu 